PROCEEDINGS OF THE

AMERICAN MATHEMATICAL SOCIETY

Volume 139, Number 7, July 2011, Pages 2287-2293

S 0002-9939(2011)10606-3

Article electronically published on March 7, 2011

\title{
HOMEOMORPHISMS OF TWO-POINT SETS
}

\author{
BEN CHAD AND CHRIS GOOD
}

(Communicated by Alexander N. Dranishnikov)

\begin{abstract}
Given a cardinal $\kappa \leq \mathfrak{c}$, a subset of the plane is said to be a $\kappa$-point set if and only if it meets every line in precisely $\kappa$ many points. In response to a question of Cobb, we show that for all $2 \leq \kappa, \lambda<\mathfrak{c}$ there exists a $\kappa$-point set which is homeomorphic to a $\lambda$-point set, and further, we also show that it is consistent with ZFC that for all $2 \leq \kappa<\mathfrak{c}$, there exists a $\kappa$-point set $X$ such that for all $2 \leq \lambda<\mathfrak{c}, X$ is homeomorphic to a $\lambda$-point set. On the other hand, we prove that it is consistent with ZFC that for all $2 \leq \kappa, \lambda<\mathfrak{c}$, there exists a $\kappa$-point set, such that for all homeomorphisms $f: \mathbb{R}^{2} \rightarrow \mathbb{R}^{2}$, if $f(X)$ is a $\lambda$-point set, then $\lambda=\kappa$.
\end{abstract}

\section{INTRODUCTION}

Given a cardinal $\kappa \leq \mathfrak{c}$, a subset of the plane is a $\kappa$-point set 11 if and only if it meets every line in precisely $\kappa$ many points, and is said to be a partial $\kappa$-point set if and only if it meets every line in at most $\kappa$ many points. By considering infinite families of concentric circles, it is easily seen that $\kappa$-point sets exist for $\aleph_{0} \leq \kappa \leq \mathfrak{c}$, and it is obvious that one-point sets do not exist. However, to demonstrate the existence of $n$-point sets for $2 \leq n<\aleph_{0}$, it seems apparent that we must resort to transfinite techniques. The standard approach, which we take in this paper, is essentially due to Mazurkiewict2 13 and is based on the existence of a wellordering of the real line, but we note that Chad et al. 3 describe an alternative construction of two-point sets which is consistent with ZF and requires only that some suitable fragment of the real line be well-ordered.

It is arguable that problems concerning two-point sets were first widely advertised amongst topologists by Mauldin 10 in his article of problems for "Open Problems in Topology" 14. Mauldin gave three problems concerning two-point sets, and to this day, the only remaining problem is to determine if a two-point set can be chosen to be a Borel subset of the plane 3 This problem is apparently very

Received by the editors October 2, 2009 and, in revised form March 31, 2010.

2010 Mathematics Subject Classification. Primary 05A99, 51N99, 54G99, 54H15.

The authors thank Rolf Suabedissen for his helpful suggestion, which led to our main result in Section 3 They thank Robin Knight for his advice concerning properties of c. And they thank an anonymous referee for helpful comments concerning an earlier draft of this paper.

${ }^{1}$ We also allow ourselves to refer to (partial) two-point sets, (partial) three-point sets, and so on.

${ }^{2}$ A French translation [12] of Mazurkiewicz's paper is available.

${ }^{3}$ Mauldin says in 11 that he "believes" he first heard of the problem from Erdös, who in turn said that it had been around since he (Erdös) was a "baby."

(C)2011 American Mathematical Society Reverts to public domain 28 years from publication 
deep, and it is likely that if we are to make any progress on it, then we will need to further our knowledge about the structure of two-point sets.

There are interesting results that are known about the structure of $\kappa$-point sets which demonstrate that differing values of $\kappa$ give rise to related but distinct classes of topological objects. For example, it is known that $n$-point sets are not $F_{\sigma}$ subsets of the plane for $2 \leq n<\aleph_{0}$, the case of $n=2$ originally having been shown by Larman [9, with corrections later supplied by Baston and Bostock 11, and the more general case having been shown by Bouhjar et al. 2. On the other hand, Larman [9] showed that two-point sets cannot contain arcs, and whilst Bouhjar et al. 22 showed that three-point sets are also required to have this property, they further showed that four-point sets are not. As continuing evidence of our claim, Kulesza [8] showed that two-point sets must be zero-dimensional, Fearnley et al. [7] showed that three-point sets must also be zero-dimensional, but the work of Bouhjar et al. 2] leads to the result that four-point sets may be either zero-dimensional or one-dimensional.

This paper investigates some general relationships which hold between the classes of $\kappa$-point sets. Our first main result can be seen to be saying that the classes of $\kappa$-point sets are pairwise overlapping (up to homeomorphism), and our second main result shows that it is consistent with ZFC that the intersection of these classes over all $2 \leq \kappa<\mathfrak{c}$ is non-empty (up to homeomorphism). Our final main result shows that it is consistent with ZFC that for all $2 \leq \kappa<\mathfrak{c}$ there exists a $\kappa$-point set $X$ such that if $f: \mathbb{R}^{2} \rightarrow \mathbb{R}^{2}$ is a homeomorphism, then $f(X)$ is either a $\kappa$-point set or it is disjoint from some line.

Our analysis has two motivations. First, given his result that there exists an $n$-point set which is homeomorphic to a function from $\mathbb{R}$ to $\mathbb{R}$ for $2 \leq n<\aleph_{0}$, Cobb [6] asks if there exists an $n$-point set which is homeomorphic to an $m$-point set for some distinct $2 \leq n, m<\aleph_{0}$. A corollary to our first main result gives an affirmative answer to Cobb's question.

The second motivation for our work is a desire to better understand the structure of two-point sets and to follow a line of research started by Chad and Suabedissen 4, 5. These papers have studied autohomeomorphims of two-point sets, and their main results include the facts that two-point sets may be chosen to be rigid or homogeneous or to have isometry group isomorphic to any subgroup of $S^{1}$ of cardinality less than $\mathfrak{c}$. These results examine a two-point set by looking for similarity within itself; our results will examine a two-point set by looking for similarity with other distinct types of geometric objects.

Throughout, we let $\mathcal{L}$ denote the collection of all lines in the plane. Also, if $2 \leq \kappa<\mathfrak{c}$, if $P$ is a partial $\kappa$-point set, and if $f$ is a homeomorphism of the plane, then we let

$$
\mathcal{L}(P, \kappa)=\{L \in \mathcal{L}:|P \cap L|=\kappa\} \quad \text { and } \quad \mathcal{L}_{f}(P, \kappa)=\{A \in f(\mathcal{L}):|P \cap A|=\kappa\} .
$$

\section{HomeOMORPHISMS OF $\kappa$-POINT SETS}

We begin by answering Cobb's question.

Lemma 2.1. There exists a family $\left\{f_{t}: t \in[0,1]\right\}$ of distinct homeomorphisms of $\mathbb{R}^{2}$ such that:

(1) $f_{0}$ is the identity function;

(2) $f_{s}(\mathcal{L}) \cap f_{t}(\mathcal{L})=\emptyset$ for all distinct $s, t \in[0,1]$; 
(3) $|A \cap B| \leq \aleph_{0}$ for all distinct $A, B \in \bigcup_{t \in[0,1]} f_{t}(\mathcal{L})$.

Proof. We find it convenient throughout to identify $\mathbb{R}^{2}$ with $\mathbb{C}$ in the usual way.

For each $r \geq 0$, let $\lfloor r\rfloor$ denote the integer part of $r$ and let $r^{\prime}=r-\lfloor r\rfloor$, so that $r^{\prime}$ denotes the fractional part of $r$. Let $g:[0,1] \rightarrow[0,1]$ be defined by $g(x)=1-|2 x-1|$, and for each $t \in[0,1]$, let $f_{t}: \mathbb{C} \rightarrow \mathbb{C}$ be the homeomorphism defined by $f_{t}\left(r e^{i \theta}\right)=r e^{i\left(\theta+t g\left(r^{\prime}\right) \pi\right)}$. We note then that $f_{0}$ is the identity function. For each $r \geq 0$, let $C_{r}$ denote the (possibly degenerate) circle centered at the origin of radius $r$. Then each $f_{t}$ rotates each $C_{r}$ by a factor of $t g\left(r^{\prime}\right) \pi$ and so leaves it invariant.

Since each $f_{s}$ and $f_{t}$ are homeomorphisms such that $f_{s}^{-1} \circ f_{t}=f_{t-s}$, it suffices to show that for all $t \in(0,1], \mathcal{L} \cap f_{t}(\mathcal{L})=\emptyset$ and that for all distinct $A, B \in \mathcal{L} \cup f_{t}(\mathcal{L})$, $|A \cap B| \leq \aleph_{0}$.

Let $t \in(0,1]$. Noting that $g^{-1}(\{0\})=\{0,1\}$, we see that $f_{t}$ fixes every point of $C_{r}$ precisely when $r \in \mathbb{Z}$ and moves every point of $C_{r}$ precisely when $r \notin \mathbb{Z}$, and so $\mathcal{L} \cap f_{t}(\mathcal{L})=\emptyset$.

Let $A, B \in \mathcal{L} \cup f_{t}(\mathcal{L})$ be distinct. If $A, B \in \mathcal{L}$, then $|A \cap B| \leq 1$, as must also be the case if $A, B \in f_{t}(\mathcal{L})$. To complete the proof, let $A=L$ and $B=f_{t}(K)$ for some $L, K \in \mathcal{L}$. To see that $|A \cap B| \leq \aleph_{0}$, it is enough to note that if $r \geq 0$ and if $S \subseteq K$ is a line segment contained in the connected region enclosed by $C_{r}$ and $C_{r+1}$, then $f_{t}(S)$ is a path which meets every line in at most four points.

Theorem 2.2. Let $2 \leq \kappa, \lambda<\mathfrak{c}$. Then there exists a $\kappa$-point set which is homeomorphic to a $\lambda$-point set.

Proof. Let $f: \mathbb{R}^{2} \rightarrow \mathbb{R}^{2}$ be a homeomorphism such that $\mathcal{L} \cap f(\mathcal{L})=\emptyset$ and distinct members of $\mathcal{L} \cup f(\mathcal{L})$ meet in at most countably many points (for example, take $f$ to be the homeomorphism $f_{1}$ furnished by Lemma 2.1), and let $\left\langle A_{\alpha}: \alpha<\mathfrak{c}\right\rangle$ be an enumeration of $\mathcal{L} \cup f(\mathcal{L})$.

We will construct an increasing sequence $\left\langle X_{\alpha}: \alpha<\mathfrak{c}\right\rangle$ of partial $\kappa$-point sets such that for all $\alpha<\mathfrak{c}$ :

(1) $X_{\alpha} \backslash \bigcup_{\beta<\alpha} X_{\beta} \subseteq A_{\alpha} \backslash\left(\bigcup \mathcal{L}\left(\bigcup_{\beta<\alpha} X_{\beta}, \kappa\right) \cup \cup \mathcal{L}_{f}\left(\bigcup_{\beta<\alpha} X_{\beta}, \lambda\right)\right)$;

(2) $X_{\alpha}$ meets each member of $\mathcal{L}$ in at most $\kappa$ many points and each member of $\mathcal{L} \cap\left\{A_{\beta}: \beta \leq \alpha\right\}$ in precisely $\kappa$ many points;

(3) $X_{\alpha}$ meets each member of $f(\mathcal{L})$ in at most $\lambda$ many points and each member of $f(\mathcal{L}) \cap\left\{A_{\beta}: \beta \leq \alpha\right\}$ in precisely $\lambda$ many points.

Suppose then that for some $\alpha<\mathfrak{c}$ we have already chosen the partial sequence $\left\langle X_{\beta}: \beta<\alpha\right\rangle$. Let $P=\bigcup_{\beta<\alpha} X_{\beta}$. Then our hypotheses imply that $|P|<\mathfrak{c}$. Let $\mu<\mathfrak{c}$ be the unique cardinal number such that

$$
\left|P \cap A_{\alpha}\right|+\mu= \begin{cases}\kappa & \text { if } A_{\alpha} \in \mathcal{L}, \\ \lambda & \text { if } A_{\alpha} \in f(\mathcal{L}) .\end{cases}
$$

If $\mu=0$, then let $X_{\alpha}=P$. Otherwise, we will select $X_{\alpha}$ in a recursion of length $\mu$. Let the sequence $\left\langle x_{\delta}: \delta<\mu\right\rangle$ be chosen such that

$x_{\delta} \in A_{\alpha} \backslash\left(\bigcup \mathcal{L}\left(P \cup\left\{x_{\gamma}: \gamma<\delta\right\}, \kappa\right) \cup \bigcup \mathcal{L}_{f}\left(P \cup\left\{x_{\gamma}: \gamma<\delta\right\}, \lambda\right) \cup\left\{x_{\gamma}: \gamma<\delta\right\}\right)$.

To confirm that such a sequence exists, we note that each member of $\mathcal{L}$ is uniquely defined by two points on it, that

$$
\left|\mathcal{L}\left(P \cup\left\{x_{\gamma}: \gamma<\delta\right\}, \kappa\right)\right|<\mathfrak{c} \text { and }\left|\mathcal{L}_{f}\left(P \cup\left\{x_{\gamma}: \gamma<\delta\right\}, \lambda\right)\right|<\mathfrak{c},
$$


and that $A_{\alpha}$ cannot be covered by fewer than $\mathfrak{c}$ many members of $(\mathcal{L} \cup f(\mathcal{L})) \backslash\left\{A_{\alpha}\right\}$. We then set $X_{\alpha}=P \cup\left\{x_{\delta}: \delta<\mu\right\}$, and since $\left\langle x_{\delta}: \delta<\mu\right\rangle$ is injective, it follows that $\left|X_{\alpha} \cap A_{\alpha}\right|$ is the required cardinal taken from $\{\kappa, \lambda\}$.

Let the $X_{\alpha}$ now be defined for all $\alpha<\mathfrak{c}$ and let $X=\bigcup_{\alpha<\mathfrak{c}} X_{\alpha}$. Conditions (2) and (3) in our recursion were chosen so that $X$ meets each member of $\mathcal{L}$ in at least $\kappa$ many points and each member of $f(\mathcal{L})$ in at least $\lambda$ many points. In the case that $\kappa$ is finite, condition (2) is sufficient to ensure that $X$ meets each member of $\mathcal{L}$ in at most $\kappa$ many points; however, in the case that $\kappa$ is infinite, we must appeal to conditions (11) and (2) to guarantee this. Similarly, it can be argued that $X$ meets each member of $f(\mathcal{L})$ in at most $\lambda$ many points. Then $f^{-1} \mid X: X \rightarrow f^{-1}(X)$ is a homeomorphism between the $\kappa$-point set $X$ and the $\lambda$-point set $f^{-1}(X)$.

The following corollary answers Cobb's question.

Corollary 2.3. Let $2 \leq n, m<\aleph_{0}$. Then there exists an $n$-point set which is homeomorphic to an m-point set.

We remark that there exists a homeomorphism $f: \mathbb{R}^{2} \rightarrow \mathbb{R}^{2}$ such that $f(X)$ is not a $\kappa$-point set for all two-point sets $X$ and all $\aleph_{0} \leq \kappa<\mathfrak{c}$. Let $H$ denote the upper half plane (including the real axis) and let $f$ be defined by

$$
f(x, y)= \begin{cases}(x, y) & \text { if } 0 \leq y \\ (x+y, y) & \text { if } y \leq 0 .\end{cases}
$$

Then $f$ has the properties that:

(1) $f$ is the identity on $H$ (and so maps line segments in $H$ to line segments in $H)$;

(2) $f\left(\mathbb{R}^{2} \backslash H\right)=\mathbb{R}^{2} \backslash H$ and maps line segments in $\mathbb{R}^{2} \backslash H$ to line segments in $\mathbb{R}^{2} \backslash H$

(3) if $L$ is a line, then there exist lines $A$ and $B$ such that $L \subseteq f(A) \cup f(B)$;

(4) $f(X)$ is a partial $2 n$-point set, and hence not a $\kappa$-point set, for all $2 \leq n<$ $\aleph_{0}$, all $\aleph_{0} \leq \kappa<\mathfrak{c}$, and all $n$-point sets $X$.

\section{The EXISTEnCE OF UNIVERSAL TWO-POINT SETS}

It turns out that we can strengthen Theorem 2.2. provided that we assume that $\mathfrak{c}$ is regular and that there are fewer than $\mathfrak{c}$ many cardinals less than $\mathfrak{c}$. These assumptions are consistent with ZFC, for they hold in models of ZFC $+\mathrm{CH}$.

Definition 3.1. Let $2 \leq \kappa<\mathfrak{c}$. Then a $\kappa$-point set is said to be universal if it is homeomorphic to a $\lambda$-point set for all $2 \leq \lambda<\mathfrak{c}$.

To prove the existence of universal $\kappa$-point sets, it will be sufficient to demonstrate the existence of a universal two-point set.

Theorem 3.2 ( $\mathfrak{c}$ is regular and $\left.\mathfrak{c}<\aleph_{\mathfrak{c}}\right)$. There exists a universal two-point set.

Proof. Let $\mu<\mathfrak{c}$ be a cardinal admitting an enumeration $\left\langle\kappa_{\gamma}: \gamma<\mu\right\rangle$ of the set of all cardinals $2<\kappa<\mathfrak{c}$, let $\left\langle t_{\gamma}: \gamma<\mu\right\rangle$ be an injective sequence on $(0,1]$, let $\left\{f_{t}: t \in[0,1]\right\}$ be a family of functions given by Lemma 2.1, let $f_{\gamma}$ denote $f_{t_{\gamma}}$, and let $\left\{A_{\alpha}: \alpha<\mathfrak{c}\right\}$ enumerate $\mathcal{L} \cup \bigcup_{\gamma<\mu} f_{\gamma}(\mathcal{L})$.

We will construct an increasing sequence $\left\langle X_{\alpha}: \alpha<\mathfrak{c}\right\rangle$ of subsets of the plane such that for all $\alpha<\mathfrak{c}$ : 
(1) $X_{\alpha} \backslash \bigcup_{\beta<\alpha} X_{\beta} \subseteq A_{\alpha} \backslash\left(\bigcup \mathcal{L}\left(\bigcup_{\beta<\alpha} X_{\beta}, 2\right) \cup \bigcup_{\gamma<\mu} \cup \mathcal{L}_{f_{\gamma}}\left(\bigcup_{\beta<\alpha} X_{\beta}, \kappa_{\gamma}\right)\right)$;

(2) $X_{\alpha}$ meets each member of $\mathcal{L}$ in at most two points and each member of $\mathcal{L} \cap\left\{A_{\beta}: \beta \leq \alpha\right\}$ in precisely two points;

(3) for each $\gamma<\mu, X_{\alpha}$ meets each member of $f_{\gamma}(\mathcal{L})$ in at most $\kappa_{\gamma}$ many points and each member of $f_{\gamma}(\mathcal{L}) \cap\left\{A_{\beta}: \beta \leq \alpha\right\}$ in precisely $\kappa_{\gamma}$ many points.

Suppose that for some $\alpha<\mathfrak{c}$ we have already chosen the partial sequence $\left\langle X_{\beta}: \beta<\alpha\right\rangle$. Let $P=\bigcup_{\beta<\alpha} X_{\beta}$. Then $\left|X_{\beta} \backslash \bigcup_{\gamma<\beta} X_{\gamma}\right|<\mathfrak{c}$ for all $\beta<\alpha$, and since $\mathfrak{c}$ is regular, it follows that $|P|<\mathfrak{c}$. We choose $X_{\alpha}$ more or less as we did in the proof of Theorem 2.2 if $A_{\alpha} \in \mathcal{L}$, then we choose $X_{\alpha}$ so that $\left|X_{\alpha} \backslash P\right| \leq 2$ and $X_{\alpha}$ meets $A_{\alpha}$ in precisely two points; otherwise, $A_{\alpha} \in f_{\gamma}(\mathcal{L})$ for some $\gamma<\mu$, and we choose $X_{\alpha}$ so that $\left|X_{\alpha} \backslash P\right| \leq \kappa_{\gamma}$ and $X_{\alpha}$ meets $A_{\alpha}$ in precisely $\kappa_{\gamma}$ many points.

Let $X=\bigcup_{\alpha<\mathfrak{c}} X_{\alpha}$. Then $X$ is a two-point set and for any $\kappa<\mathfrak{c}, \kappa=\kappa_{\gamma}$ for some $\gamma<\mu$, and $f_{\gamma}^{-1}(X)$ is a $\kappa$-point set homeomorphic to $X$.

Corollary $3.3\left(\mathfrak{c}\right.$ is regular and $\left.\mathfrak{c}<\aleph_{\mathfrak{c}}\right)$. Let $2 \leq \kappa \leq \aleph_{0}$. Then there exists a universal $\kappa$-point set.

\section{The EXISTEnCE OF DELICATE $\kappa$-POINT SETS}

We will now consider how $\kappa$-point sets are embedded in the plane and obtain a result which is complementary to those we have previously discussed. We will make use of the axiom that " $\mathbb{R}$ cannot be covered by fewer than $\mathfrak{c}$ many of its nowhere dense subsets", and we will refer to this axiom, which is implied by the Continuum Hypothesis or Martin's Axiom, by $\operatorname{cov}(\mathcal{M})=\mathfrak{c}$.

Definition 4.1. Let $2 \leq \kappa<\mathfrak{c}$. Then a $\kappa$-point set $X$ is said to be delicate if for every homeomorphism $f: \mathbb{R}^{2} \rightarrow \mathbb{R}^{2}$, if $f$ is not affine, then $f(X)$ is disjoint from some line.

Lemma 4.2. Let $f: \mathbb{R}^{2} \rightarrow \mathbb{R}^{2}$ be a non-affine homeomorphism. Then there exists a collection $\mathcal{K}$ of lines such that:

(1) $f^{-1}(K)$ is not a line for each $K \in \mathcal{K}$;

(2) the members of $\mathcal{K}$ are pairwise parallel;

(3) $|\mathcal{K}|=\mathfrak{c}$.

Proof. Note that a bijection $\mathbb{R}^{2} \rightarrow \mathbb{R}^{2}$ is affine if and only if its inverse is affine. Let $x, y, z \in \mathbb{R}^{2}$ be collinear and such that $f^{-1}(x), f^{-1}(y)$ and $f^{-1}(z)$ form the vertices of a non-degenerate triangle $T$. Let $\varepsilon_{1}>0$ be the length of the altitude of $T$ joining $f^{-1}(z)$ to the line segment joining $f^{-1}(x)$ and $f^{-1}(y)$, let $\varepsilon_{2}>0$ be the distance between $f^{-1}(x)$ and $f^{-1}(y)$, and let $\varepsilon=\min \left\{\varepsilon_{1} / 2, \varepsilon_{2} / 2\right\}$. Then letting $B(v, r)$ denote the ball of radius $r>0$ about $v \in \mathbb{R}^{2}$, it is easily seen that $B\left(f^{-1}(x), \varepsilon\right)$, $B\left(f^{-1}(y), \varepsilon\right)$ and $B\left(f^{-1}(z), \varepsilon\right)$ are pairwise disjoint. Further, it can be seen that for all $a \in B\left(f^{-1}(x), \varepsilon\right)$, all $b \in B\left(f^{-1}(y), \varepsilon\right)$ and all $c \in B\left(f^{-1}(z), \varepsilon\right)$, the points $a$, $b$ and $c$ form the vertices of a non-degenerate triangle.

Given that $f^{-1}$ is continuous, let $\delta>0$ be such that $f^{-1}(B(x, \delta)) \subseteq B\left(f^{-1}(x), \varepsilon\right)$ and $f^{-1}(B(y, \delta)) \subseteq B\left(f^{-1}(y), \varepsilon\right)$ and $f^{-1}(B(z, \delta)) \subseteq B\left(f^{-1}(z), \varepsilon\right)$. Let $L$ be the line spanned by $x$ and $y$, let $v \in \mathbb{R}^{2}$ be of unit length and perpendicular to $L$, and let $\mathcal{K}=\{L+r v: r \in[0, \delta)\}$. Then it is clear that the members of $\mathcal{K}$ are pairwise parallel and $|K|=\mathfrak{c}$. Further, for each $r \in[0, \delta)$, the points $f^{-1}(x+r v), f^{-1}(y+r v)$ and $f^{-1}(z+r v)$ witness that $f^{-1}(L+r v)$ is not a line. 
Theorem $4.3(\operatorname{cov}(\mathcal{M})=\mathfrak{c})$. Let $2 \leq \kappa<\mathfrak{c}$. Then there exists a delicate $\kappa$-point set.

Proof. Let $2 \leq \kappa<\mathfrak{c}$, let $\left\langle L_{\alpha}: \alpha<\mathfrak{c}\right\rangle$ enumerate the collection of all lines, and let $\left\langle f_{\alpha}: \alpha<\mathfrak{c}\right\rangle$ enumerate all non-affine autohomeomorphims of $\mathbb{R}^{2}$. By the previous lemma, let $\left\langle\mathcal{K}_{\alpha}: \alpha<\mathfrak{c}\right\rangle$ be a sequence of collections of lines such that for each $\alpha<\mathfrak{c}$ :

(a) for each $K \in \mathcal{K}_{\alpha}, f_{\alpha}^{-1}(K)$ is not a line;

(b) the members of $\mathcal{K}_{\alpha}$ are pairwise parallel;

(c) $\left|\mathcal{K}_{\alpha}\right|=\mathfrak{c}$.

We note then that the members of each $\mathcal{K}_{\alpha}$ are pairwise disjoint, as are the members of each $f_{\alpha}^{-1}\left(\mathcal{K}_{\alpha}\right)$.

We will construct an increasing sequence $\left\langle X_{\alpha}: \alpha<\mathfrak{c}\right\rangle$ of partial $\kappa$-point sets and a sequence $\left\langle K_{\alpha}: \alpha<\mathfrak{c}\right\rangle$ of lines such that for all $\alpha<\mathfrak{c}$ :

(1) $\left|X_{\alpha} \backslash \bigcup_{\beta<\alpha} X_{\beta}\right| \leq \kappa+\aleph_{0}$ and $\left(X_{\alpha} \backslash \bigcup_{\beta<\alpha} X_{\beta}\right) \cap \bigcup \mathcal{L}\left(\bigcup_{\beta<\alpha} X_{\beta}, \kappa\right)=\emptyset$;

(2) $X_{\alpha}$ meets each member of $\mathcal{L}$ in at most $\kappa$ many points and each member of $\left\{L_{\beta}: \beta \leq \alpha\right\}$ in precisely $\kappa$ many points;

(3) $K_{\alpha} \in \mathcal{K}_{\alpha}$;

(4) $X_{\alpha}$ and $\bigcup_{\beta \leq \alpha} f_{\beta}^{-1}\left(K_{\beta}\right)$ are disjoint;

(5) for each $\beta<\alpha$ and for each $\gamma<\mathfrak{c}$, if $\left|X_{\beta} \cap L_{\gamma}\right|<\kappa$, then $f_{\beta}^{-1}\left(K_{\beta}\right) \cap L_{\gamma}$ is nowhere dense in the relative topology on $L_{\gamma}$.

Suppose then that for some $\alpha<\mathfrak{c}$ we have already chosen the partial sequences $\left\langle X_{\beta}: \beta<\alpha\right\rangle$ and $\left\langle K_{\beta}: \beta<\alpha\right\rangle$. Let $P=\bigcup_{\beta<\alpha} X_{\beta}$ and let $F=\bigcup_{\beta<\alpha} f_{\beta}^{-1}\left(K_{\beta}\right)$. We will choose $X_{\alpha}$ in two steps. First, we will extend $P$ to $Q$ so as to satisfy conditions (1) - (4), and then we will extend $Q$ to $X_{\alpha}$ so as to preserve these properties and to additionally satisfy condition (5).

If $\left|P \cap L_{\alpha}\right|=\kappa$, then let $Q=P$. Otherwise it follows from the inductive hypothesis and $\operatorname{cov}(\mathcal{M})=\mathfrak{c}$ that $\left|L_{\alpha} \backslash F\right|=\mathfrak{c}$, and so by choosing suitable points in

$$
L_{\alpha} \backslash(\bigcup \mathcal{L}(P, \kappa) \cup F),
$$

let $Q$ be a partial $\kappa$-point set such that $P \subseteq Q$ and $Q \backslash P \subseteq L_{\alpha}$ and $\left|L_{\alpha} \cap Q\right|=\kappa$.

It is easily argued from (1) that $|Q|<\mathfrak{c}$, and so we may choose $K_{\alpha} \in \mathcal{K}_{\alpha}$ to be such that $Q \cap f_{\alpha}^{-1}\left(K_{\alpha}\right)=\emptyset$. Let

$$
\mathcal{A}=\left\{L \in \mathcal{L}:|Q \cap L|<\kappa \text { and } f_{\alpha}^{-1}\left(K_{\alpha}\right) \cap L \text { is somewhere dense in } L\right\} .
$$

We will now argue that $\mathcal{A}$ is countable. Towards this, let

$$
\mathcal{S}=\left\{S \subseteq f_{\alpha}^{-1}\left(K_{\alpha}\right): S \text { is a maximal non-degenerate line segment }\right\},
$$

and so noting that $L \in \mathcal{A}$ if and only if $L \in \mathcal{L}$ and $L$ contains a member of $S$, it will suffice to show that $\mathcal{S}$ is countable. This is obvious once we note that a maximal non-degenerate line segment contained in $f_{\alpha}^{-1}\left(K_{\alpha}\right)$ is the image under $f_{\alpha}^{-1}$ of some interval contained in $K_{\alpha}$.

It now remains to describe how to extend $Q$ to $X_{\alpha}$ so that $X_{\alpha}$ meets each member of $\mathcal{A}$ in precisely $\kappa$ many points. For each $L \in \mathcal{A}$ it is a routine exercise to show that $L \backslash f_{\alpha}^{-1}\left(K_{\alpha}\right) \neq \emptyset$, and so we can choose $x_{L} \in L \backslash f_{\alpha}^{-1}\left(K_{\alpha}\right)$. Since $\mathbb{R}^{2} \backslash f_{\alpha}^{-1}\left(K_{\alpha}\right)$ is an open neighbourhood of $x_{L}$, it follows that there exists an interval $I_{L} \subseteq L \backslash f_{\alpha}^{-1}\left(K_{\alpha}\right)$. 
By choosing suitable points in

$$
\left(\bigcup_{L \in \mathcal{A}} I_{L}\right) \backslash\left(\bigcup \mathcal{L}(Q, \kappa) \cup F \cup f_{\alpha}^{-1}\left(K_{\alpha}\right)\right),
$$

we select $X_{\alpha}$ to be a partial $\kappa$-point set which extends $Q$ and meets each $L \in \mathcal{A}$ in $\kappa$ many points. Then $X_{\alpha}$ and $K_{\alpha}$ satisfy our inductive hypothesis.

Let $X=\bigcup_{\alpha<\mathfrak{c}} X_{\alpha}$. Then $X$ is a $\kappa$-point set. To see that $X$ is delicate, it suffices to note that if $f: \mathbb{R}^{2} \rightarrow \mathbb{R}^{2}$ is a non-affine homeomorphism, then $f=f_{\alpha}$ for some $\alpha<\mathfrak{c}$, whence $f(X)=f_{\alpha}(X)$ is disjoint from the line $K_{\alpha}$.

Corollary $4.4(\operatorname{cov}(\mathcal{M})=\mathfrak{c})$. There exists a delicate two-point set.

Note that if $2 \leq \kappa, \lambda<\mathfrak{c}$ and $X$ is a delicate $\kappa$-point set and $f: \mathbb{R}^{2} \rightarrow \mathbb{R}^{2}$ is a homeomorphism, then whenever $f(X)$ is a $\lambda$-point set we must have that $\lambda=\kappa$.

\section{REFERENCES}

[1] V. J. Baston and F. A. Bostock. On a theorem of Larman. J. London Math. Soc. (2), 5:715718, 1972. MR0315589(47:4138)

[2] Khalid Bouhjar, Jan J. Dijkstra, and Jan van Mill. Three-point sets. Topology Appl., 112(2):215-227, 2001. MR.1823606 (2002c:54034)

[3] Ben Chad, Robin Knight, and Rolf Suabedissen. Set-theoretic constructions of two-point sets. Fund. Math., 203(2):179-189, 2009. MR2496237(2009m:03077)

[4] Ben Chad and Rolf Suabedissen. Symmetries of two-point sets. Topology Appl., 155(11):12131220, 2008. MR2421831 (2009f:54049)

[5] Ben Chad and Rolf Suabedissen. On affine groups admitting invariant two-point sets. Topology Appl., 156(13):2209-2213, 2009. MR.2544128 (2010f:54051)

[6] John Cobb. $n$-point sets and graphs of functions. Topology Appl., 155(16):1804-1807, 2008. MR2445304 (2010a:54052)

[7] David L. Fearnley, L. Fearnley, and J. W. Lamoreaux. Every three-point set is zero dimensional. Proc. Amer. Math. Soc., 131(7):2241-2245 (electronic), 2003. MR.1963773 (2004b:51022)

[8] John Kulesza. A two-point set must be zero-dimensional. Proc. Amer. Math. Soc., 116(2):551553, 1992. MR1093599 (92m:54064)

[9] D. G. Larman. A problem of incidence. J. London Math. Soc., 43:407-409, 1968. MR0231724 (38:52)

[10] R. Daniel Mauldin. Problems in topology arising from analysis. In Open problems in topology, pages 617-629. North-Holland, Amsterdam, 1990. MR.1078668

[11] R. Daniel Mauldin. On sets which meet each line in exactly two points. Bull. London Math. Soc., 30(4):397-403, 1998. MR1620829 (99f:28010)

[12] S. Mazurkiewicz. O pewnej mnogości plaskiej, która ma z każda prosta dwa i tylko dwa punkty wspólne. C. R. Varsovie, 7:382-384, 1914.

[13] Stefan Mazurkiewicz. Travaux de topologie et ses applications. Comité de rédaction: K. Borsuk, R. Engelking, B. Knaster, K. Kuratowski, J. Loś, R. Sikorski. Państwowe Wydawnictwo Naukowe-Éditions Scientifiques de Pologne, Warsaw, 1969. MR 0250248 (40:3487)

[14] Jan van Mill and George M. Reed, editors. Open problems in topology. North-Holland Publishing Co., Amsterdam, 1990. MR1078636 (92c:54001)

St Edmund Hall, University of Oxford, Oxford, OX1 4AR, United Kingdom

E-mail address: chad@maths.ox.ac.uk

School of Mathematics and Statistics, The University of Birmingham, Edgbaston, Birmingham, B15 2TT, United Kingdom

E-mail address: c.good@bham.ac.uk 